\title{
Topological degree and applications to elliptic problems with discontinuous nonlinearity
}

In-Sook Kim

Department of Mathematics, Sungkyunkwan University, Suwon 16419, Republic of Korea.

Communicated by Y. J. Cho

\begin{abstract}
We develop a topological degree theory for a class of locally bounded weakly upper semicontinuous set-valued operators of generalized $\left(\mathrm{S}_{+}\right)$type in real reflexive separable Banach spaces, based on the Berkovits-Tienari degree. The method of approach is to use elliptic super-regularization by means of certain compact embeddings, instead of the Galerkin method. Applying the degree theory, we tackle an elliptic boundary value problem with discontinuous nonlinearity. (C)2017 All rights reserved.
\end{abstract}

Keywords: Set-valued operators of $\left(S_{+}\right)$type, degree theory, $p$-Laplacian.

2010 MSC: 47H04, 47H05, 47H11, 47H30, 47J05.

\section{Introduction and preliminaries}

Topological degree theory has been widely used in the study of nonlinear equations. Leray and Schauder [10] introduced a degree theory for compact perturbations of the identity in infinite-dimensional Banach spaces. Browder [4] constructed a topological degree for operators of class $\left(S_{+}\right)$in reflexive Banach spaces with the Galerkin method, see also [12, 14, 15]. Berkovits [2] considered an extension of the classical Leray-Schauder degree for operators of generalized monotone type. Roughly speaking, the class of operators for the extended degree is essentially obtained by replacing the compact perturbation by a composition of operators of monotone type.

We consider in a real reflexive Banach space a nonlinear inclusion of the form

$$
h \in \mathrm{Fu},
$$

where $F$ is a bounded weakly upper semicontinuous set-valued operator of generalized $\left(S_{+}\right)$type. Berkovits and Tienari [3] developed a topological degree for bounded weakly upper semicontinuous operators of class $\left(S_{+}\right)$based on the known degree for Leray-Schauder type set-valued operators given in $[7,11]$.

In the present paper, the goal is to study a topological degree theory for a wider class of locally bounded weakly upper semicontinuous set-valued operators of generalized $\left(S_{+}\right)$type in real reflexive

Email address: iskim@skku.edu (In-Sook Kim)

doi:10.22436/jnsa.010.02.24 
separable Banach spaces. This may be regarded as a set-valued version of the degree theory for bounded demicontinuous operators of generalized $\left(S_{+}\right)$type due to Berkovits [2], see also [9]. In order to construct a new degree, we first observe that a locally bounded operator can be reduced to some bounded operator on a suitable domain. So we proceed in two steps. Adopting elliptic super-regularization method as in [2], we can apply the Berkovits-Tienari degree theory to define a topological degree for the bounded operator case. Taking this observation into account, we then introduce a degree theory for the class of locally bounded weakly upper semicontinuous operators of generalized $\left(S_{+}\right)$type.

The class of operators considered in (1.1) contains abstract Hammerstein operators of the type $\mathrm{F}=$ $\mathrm{I}+\mathrm{ST}$, where I denotes the identity operator and $\mathrm{S}, \mathrm{T}$ are of monotone type, for instance, of class $\left(S_{+}\right)$ or quasimonotone. In many applications, the formulation of a boundary value problem gives rise to an abstract Hammerstein equation. In this aspect, we study an elliptic boundary value problem of the form

$$
\left\{\begin{array}{cl}
-\Delta_{\mathfrak{p}} u+u \in-[\underline{g}(x, u), \bar{g}(x, u)], & \text { in } \Omega, \\
u=0, & \text { on } \partial \Omega,
\end{array}\right.
$$

where $\Omega$ is a bounded domain in $\mathbb{R}^{N}, \Delta_{p}$ is the $p$-Laplacian, and $g$ is a possibly discontinuous function in the second variable. The point is that the celebrated Browder-Minty theorem on monotone operators plays a decisive role in transforming (1.2) into a Hammerstein equation. This enables us to find a weak solution of the given problem (1.2) using the degree theory. For related topics, we refer to [3, 8].

The paper is organized as follows. In this Section, we give some definitions and basic facts for certain classes of operators which will be needed later. In Section 2, we introduce a topological degree theory for a wider class of locally bounded weakly upper semicontinuous operators of generalized $\left(S_{+}\right)$type in real reflexive Banach spaces, based on the Berkovits-Tienari degree. Section 3 deals with the solvability of the elliptic boundary value problem with discontinuous nonlinearity.

Let $X$ be a real Banach space. Given a nonempty subset $\Omega$ of $X$, let $\bar{\Omega}$ and $\partial \Omega$ denote the closure and the boundary of $\Omega$ in $X$, respectively. Let $B_{r}(u)$ denote the open ball in $X$ of radius $r>0$ centered at $u$.

Definition 1.1. Let $Y$ be another real Banach space. A set-valued operator $F: \Omega \subset X \rightarrow 2^{Y}$ is said to be

(1) upper semicontinuous (u.s.c.), if the set $F^{-1}(A)=\{u \in \Omega \mid F u \cap A \neq \emptyset\}$ is closed in $X$ for each closed set $A$ in $Y$;

(2) weakly upper semicontinuous (w.u.s.c.), if $F^{-1}(A)$ is closed in $X$ for each weakly closed set $A$ in $Y$;

(3) compact, if it is upper semicontinuous and the image of any bounded set is relatively compact;

(4) bounded, if F maps bounded sets into bounded sets;

(5) locally bounded, if for each $u \in \Omega$ there exists a neighborhood $U$ of $u$ such that the set $F(U)=$ $\bigcup_{\mathfrak{u} \in \mathrm{U}} \mathrm{Fu}$ is bounded.

Let $X$ be a real reflexive Banach space with dual space $X^{*}$. The symbol $\langle\cdot, \cdot\rangle_{X}$ denotes the dual paring between $X^{*}$ and $X$ in this order. Identifying the bidual space $X^{* *}$ with $X$, we sometimes write $\langle y, x\rangle$ for $\langle x, y\rangle_{X^{*}}$ for $x \in X$ and $y \in X^{*}$. The symbol $\rightarrow(\rightarrow)$ stands for strong (weak) convergence.

Definition 1.2. A set-valued operator $F: \Omega \subset X \rightarrow 2^{X^{*}} \backslash \emptyset$ is said to be

(1) of class $\left(S_{+}\right)$, if for any sequence $\left(u_{n}\right)$ in $\Omega$ and any sequence $\left(w_{n}\right)$ in $X^{*}$ with $w_{n} \in F u_{n}$ such that $u_{n} \rightarrow u$ in $X$ and

we have $u_{n} \rightarrow u$ in $X$;

$$
\limsup _{n \rightarrow \infty}\left\langle w_{n}, u_{n}-u\right\rangle \leqslant 0
$$

(2) quasimonotone, if for any sequence $\left(u_{n}\right)$ in $\Omega$ and any sequence $\left(w_{n}\right)$ in $X^{*}$ with $w_{n} \in F u_{n}$ such that $u_{n} \rightarrow u$ in $X$, we have

$$
\liminf _{n \rightarrow \infty}\left\langle w_{n}, u_{n}-u\right\rangle \geqslant 0 .
$$


Definition 1.3. Let $T: \Omega_{1} \subset X \rightarrow X^{*}$ be a bounded operator such that $\Omega \subset \Omega_{1}$. A set-valued operator $F: \Omega \subset X \rightarrow 2^{X} \backslash \emptyset$ is said to be:

(1) of class $\left(S_{+}\right)_{T}$, if for any sequence $\left(u_{n}\right)$ in $\Omega$ and any sequence $\left(w_{n}\right)$ in $X$ with $w_{n} \in F u_{n}$ such that $u_{n} \rightarrow u$ in $X, T u_{n} \rightarrow y$ in $X^{*}$ and

$$
\limsup _{n \rightarrow \infty}\left\langle w_{n}, T u_{n}-y\right\rangle \leqslant 0,
$$

we have $u_{n} \rightarrow u$ in $X$;

(2) T-quasimonotone, written $F \in(Q M)_{T}$, if for any sequence $\left(u_{n}\right)$ in $\Omega$ and any sequence $\left(w_{n}\right)$ in $X$ with $w_{n} \in F u_{n}$ such that $u_{n} \rightarrow u$ in $X, T u_{n} \rightarrow y$ in $X^{*}$, we have

$$
\liminf _{n \rightarrow \infty}\left\langle w_{n}, T u_{n}-y\right\rangle \geqslant 0
$$

Notice that if $F: \Omega \subset X \rightarrow 2^{X} \backslash \emptyset$ is locally bounded and of class $\left(S_{+}\right)_{T}$, where $\Omega$ is closed in $X$ and $\mathrm{T}: \Omega \rightarrow \mathrm{X}^{*}$ is bounded and continuous, then $\mathrm{F}$ is $\mathrm{T}$-quasimonotone. Moreover, the collection of operators of class $\left(S_{+}\right)_{T}$ is stable under $(\mathrm{QM})_{\mathrm{T}}$-perturbations. See [9, Lemma 2.1] for the single-valued case.

Throughout this paper, we always assume that all weakly upper semicontinuous set-valued operators considered have nonempty closed convex values.

We consider the following classes of operators:

$$
\begin{aligned}
& \mathcal{F}_{1}(\Omega):=\left\{\mathrm{F}: \Omega \subset \mathrm{X} \rightarrow \mathrm{X}^{*} \mid \mathrm{F} \text { is bounded, continuous and of class }\left(\mathrm{S}_{+}\right)\right\}, \\
& \mathcal{F}_{\mathrm{S}_{+}}(\Omega):=\left\{\mathrm{F}: \Omega \subset \mathrm{X} \rightarrow 2^{X^{*}} \mid \mathrm{F} \text { is bounded, w.u.s.c. and of class }\left(\mathrm{S}_{+}\right)\right\}, \\
& \mathcal{F}_{\mathrm{T}, \mathrm{B}}(\Omega):=\left\{\mathrm{F}: \Omega \subset \mathrm{X} \rightarrow 2^{\mathrm{X}} \mid \mathrm{F} \text { is bounded, w.u.s.c. and of class }\left(\mathrm{S}_{+}\right)_{\mathrm{T}}\right\}, \\
& \mathcal{F}_{\mathrm{T}}(\Omega):=\left\{\mathrm{F}: \Omega \subset \mathrm{X} \rightarrow 2^{\mathrm{X}} \mid \mathrm{F} \text { is locally bounded, w.u.s.c. and of class }\left(\mathrm{S}_{+}\right)_{\mathrm{T}}\right\},
\end{aligned}
$$

for any set $\Omega \subset D_{F}$ and any bounded operator $T: \Omega \rightarrow X^{*}$, where $D_{F}$ denotes the domain of $F$.

Let

$$
\begin{aligned}
& \mathcal{F}_{S_{+}}(X):=\left\{F \in \mathcal{F}_{S_{+}}(\bar{G}) \mid G \in \mathcal{O}\right\}, \\
& \mathcal{F}_{\mathrm{B}}(X):=\left\{F \in \mathcal{F}_{T, B}(\bar{G}) \mid G \in \mathcal{O}, T \in \mathcal{F}_{1}(\bar{G})\right\}, \\
& \mathcal{F}(X):=\left\{F \in \mathcal{F}_{\mathrm{T}}(\overline{\mathrm{G}}) \mid \mathrm{G} \in \mathcal{O}, T \in \mathcal{F}_{1}(\overline{\mathrm{G}})\right\},
\end{aligned}
$$

where $\mathcal{O}$ denotes the collection of all bounded open sets in $X$. Here, $T \in \mathcal{F}_{1}(\bar{G})$ is called an essential inner map to F.

The following result shows that the Hammerstein operator of the type I+ST belongs to the class $\mathcal{F}(X)$. For the single-valued case, we refer to [2, Lemma 2.2] and [9, Lemma 2.3].

Lemma 1.4. Let $\mathrm{G}$ be any bounded open set in a real reflexive Banach space $X$. Suppose that $T \in \mathcal{F}_{1}(\overline{\mathrm{G}})$ and $\mathrm{S}: \mathrm{D}_{\mathrm{S}} \subset \mathrm{X}^{*} \rightarrow 2^{\mathrm{X}}$ is locally bounded and weakly upper semicontinuous such that $\mathrm{T}(\overline{\mathrm{G}}) \subset \mathrm{D}_{\mathrm{S}}$. Then the following statements hold:

(a) If $\mathrm{S}$ is quasimonotone, then $\mathrm{I}+\mathrm{ST} \in \mathcal{F}_{\mathrm{T}}(\overline{\mathrm{G}})$.

(b) If $S$ is of class $\left(S_{+}\right)$, then $S T \in \mathcal{F}_{T}(\bar{G})$.

Proof. (a): Set $F:=I+S T$. Let $\left(u_{k}\right)$ be any sequence in $\bar{G}$ and $\left(w_{k}\right)$ be any sequence in $X$ with $w_{k} \in S T u_{k}$ such that

$$
u_{k} \rightarrow u \text { in } X, \quad y_{k}:=T u_{k} \rightarrow y \text { in } X^{*}, \quad \text { and } \limsup _{k \rightarrow \infty}\left\langle u_{k}+w_{k}, y_{k}-y\right\rangle \leqslant 0 .
$$

Since the sequence $\left(\left\langle T u_{k}, u_{k}-\mathfrak{u}\right\rangle\right)$ is bounded in $\mathbb{R}$, there is a subsequence $\left(u_{j}\right)$ of $\left(u_{k}\right)$ such that the limit $\lim _{j \rightarrow \infty}\left\langle T u_{j}, u_{j}-u\right\rangle$ exists. By the reflexivity of $X$, we can write it in the form:

$$
\lim _{j \rightarrow \infty}\left\langle u_{j}, y_{j}-y\right\rangle_{X^{*}}=\lim _{j \rightarrow \infty}\left\langle T u_{j}, u_{j}-u\right\rangle
$$


By the quasimonotonicity of $S,(1.3)$ and (1.4), we get

$$
\begin{aligned}
0 & \leqslant \limsup _{j \rightarrow \infty}\left\langle w_{j}, y_{j}-y\right\rangle \\
& =\limsup _{j \rightarrow \infty}\left\langle u_{j}+w_{j}, y_{j}-y\right\rangle-\lim _{j \rightarrow \infty}\left\langle u_{j}, y_{j}-y\right\rangle \\
& \leqslant-\lim _{j \rightarrow \infty}\left\langle T u_{j}, u_{j}-u\right\rangle .
\end{aligned}
$$

Since $T$ is of class $\left(S_{+}\right)$, we have $u_{j} \rightarrow u$ in $X$. By the convergence principle in [16, Proposition 10.13], the entire sequence $\left(u_{k}\right)$ converges strongly to $u$ in $X$. Thus the operator $F$ is of class $\left(S_{+}\right)_{T}$. Since ST is locally bounded and weakly upper semicontinuous on $\bar{G}$, we conclude that $F \in \mathcal{F}_{\mathrm{T}}(\overline{\mathrm{G}})$.

(b): Let $\left(u_{k}\right)$ be any sequence in $\bar{G}$ and $\left(w_{k}\right)$ be any sequence in $X$ with $w_{k} \in S T u_{k}$ such that

$$
u_{k} \rightarrow u \text { in } X, \quad y_{k}:=T u_{k} \rightarrow y \text { in } X^{*}, \quad \text { and } \limsup _{k \rightarrow \infty}\left\langle w_{k}, y_{k}-y\right\rangle \leqslant 0 .
$$

Since $S$ is of class $\left(S_{+}\right)$, it follows that $y_{k} \rightarrow y$ in $X^{*}$. Since $\lim _{k \rightarrow \infty}\left\langle T u_{k}, u_{k}-\mathfrak{u}\right\rangle=0$ and $T$ is of class $\left(S_{+}\right)$, we have $u_{k} \rightarrow u$ in $X$. Consequently, we obtain that $S T \in \mathcal{F}_{T}(\bar{G})$. This completes the proof.

Actually, we see by simple examples in $[2,9]$ that in a Hilbert space the collection of operators of class $\left(S_{+}\right)_{\mathrm{T}}$ is larger than that of class $\left(S_{+}\right)$.

Definition 1.5. For a bounded operator $T: \bar{G} \subset X \rightarrow X^{*}$, a homotopy $H:[0,1] \times \bar{G} \rightarrow 2^{X}$ is said to be of class $\left(S_{+}\right)_{T}$, if for any sequence $\left(t_{k}, u_{k}\right)$ in $[0,1] \times \bar{G}$ and any sequence $\left(a_{k}\right)$ in $X$ with $a_{k} \in H\left(t_{k}, u_{k}\right)$ such that

$$
u_{k} \rightarrow \mathfrak{u} \text { in } X, \quad t_{k} \rightarrow t \text { in }[0,1], \quad T u_{k} \rightarrow y \text { in } X^{*}, \quad \text { and } \quad \limsup _{k \rightarrow \infty}\left\langle a_{k}, T u_{k}-y\right\rangle \leqslant 0,
$$

we have $u_{k} \rightarrow u$ in $X$.

The following result says that every affine homotopy with a common essential inner map $T$ is of class $\left(S_{+}\right)_{\mathrm{T}}$.

Lemma 1.6. Let $\mathrm{G}$ be a bounded open subset of a real reflexive Banach space $X$ and let $T: \bar{G} \rightarrow X^{*}$ be bounded and continuous. If $\mathrm{F}, \mathrm{S}$ are bounded and of class $\left(\mathrm{S}_{+}\right)_{\mathrm{T}}$, then an affine homotopy $\mathrm{H}:[0,1] \times \overline{\mathrm{G}} \rightarrow 2^{X}$ defined by

$$
\mathrm{H}(\mathrm{t}, \mathrm{u}):=(1-\mathrm{t}) \mathrm{Fu}+\mathrm{tSu}, \quad \text { for }(\mathrm{t}, \mathrm{u}) \in[0,1] \times \overline{\mathrm{G}},
$$

is of class $\left(\mathrm{S}_{+}\right)_{\mathrm{T}}$.

Proof. Let $\left(u_{k}\right)$ be any sequence in $\bar{G}$ and $\left(t_{k}\right)$ be any sequence in $[0,1]$ such that

$$
u_{k} \rightarrow u \text { in } X, \quad t_{k} \rightarrow t \text { in }[0,1], \quad y_{k}:=T u_{k} \rightarrow y \text { in } X^{*}, \quad \text { and } \quad \limsup _{k \rightarrow \infty}\left\langle a_{k}, y_{k}-y\right\rangle \leqslant 0,
$$

where $a_{k}=\left(1-t_{k}\right) v_{k}+t_{k} w_{k} \in H\left(t_{k}, u_{k}\right), v_{k} \in F u_{k}$ and $w_{k} \in S u_{k}$. Note that

$$
\left\langle a_{k}, y_{k}-y\right\rangle=\left(1-t_{k}\right)\left\langle v_{k}, y_{k}-y\right\rangle+t_{k}\left\langle w_{k}, y_{k}-y\right\rangle \text {. }
$$

If $t=1$, then it follows from the boundedness of $F$ that

$$
\limsup _{k \rightarrow \infty}\left\langle w_{k}, y_{k}-y\right\rangle=\limsup _{k \rightarrow \infty}\left\langle a_{k}, y_{k}-y\right\rangle \leqslant 0,
$$

which implies, in view of $S \in\left(S_{+}\right)_{T}$ that $u_{k} \rightarrow u$ in $X$. If $t \in[0,1)$, then $S \in(Q M)_{T}$ implies that

$$
\begin{aligned}
(1-t) \limsup _{k \rightarrow \infty}\left\langle v_{k}, y_{k}-y\right\rangle & \leqslant(1-t) \limsup _{k \rightarrow \infty}\left\langle v_{k}, y_{k}-y\right\rangle+t \liminf \left\langle w_{k}, y_{k}-y\right\rangle \\
& \leqslant \limsup _{k \rightarrow \infty}\left\langle a_{k}, y_{k}-y\right\rangle \leqslant 0 .
\end{aligned}
$$

Since $F$ is of class $\left(S_{+}\right)_{T}$, we have $u_{k} \rightarrow u$ in $X$. In both cases, we have shown that $u_{k} \rightarrow u$ in $X$. This 
completes the proof.

For the construction of our degree, we now observe that a locally bounded homotopy can be reduced to some bounded homotopy on a suitable domain. This is a more general set-valued version of $[9$, Theorem 2.6] under some weaker conditions.

Theorem 1.7. Let $\mathrm{G}$ be an open set in a real Banach space $\mathrm{X}$ and let $\mathrm{Y}$ be a real normed space. Suppose that $\mathrm{H}:[0,1] \times \overline{\mathrm{G}} \rightarrow 2^{\mathrm{Y}}$ is a locally bounded homotopy. If $\mathrm{S}$ is a nonempty compact set in $\mathrm{X}$ with $\mathrm{S} \subset \mathrm{G}$, then there exists an open set $\mathrm{G}_{0}$ in $\mathrm{X}$ such that

(a) $\mathrm{S} \subset \mathrm{G}_{0} \subset \mathrm{G}$, and

(b) $\|\mathrm{a}\| \leqslant \mathrm{R}$, for all $(\mathrm{t}, \mathrm{u}) \in[0,1] \times \overline{\mathrm{G}}_{0}$ and all $\mathrm{a} \in \mathrm{H}(\mathrm{t}, \mathrm{u})$ and for some positive constant $\mathrm{R}$.

Proof. Let $\mathrm{S}$ be a nonempty compact set in $\mathrm{X}$ with $\mathrm{S} \subset \mathrm{G}$ and let

$$
D_{n}:=\left\{u \in X \mid \operatorname{dist}(u, S)<\frac{1}{n}\right\}, \quad \text { for } n \in \mathbb{N} .
$$

By the compactness of $S$, it can be expressed as

$$
D_{n}=\left\{u \in X \mid\|u-z\|<\frac{1}{n} \text { for some } z \in S\right\} .
$$

Setting $G_{n}:=D_{n} \cap G$, it is clear that $G_{n}$ is open in $X$ and $S \subset G_{n} \subset G$, that is, (a) holds for each $G_{n}$. We now have to verify that at least one of the sets $G_{n}$ fulfills property (b). If none of the sets $G_{n}$ satisfies (b), there are sequences $\left(t_{n}\right)$ in $[0,1]$ and $\left(u_{n}\right)$ in $\bar{G}_{n}$ such that

$$
\left\|a_{n}\right\|>n,
$$

where $a_{n} \in H\left(t_{n}, u_{n}\right)$. According to $u_{n} \in \bar{D}_{n}$, we can choose a sequence $\left(z_{n}\right)$ in $S$ such that $\left\|u_{n}-z_{n}\right\| \leqslant$ $2 / \mathrm{n}$. Since the set $S$ is compact, there exists a subsequence $\left(z_{k}\right)$ of $\left(z_{\mathfrak{n}}\right)$ which converges to some $z \in S$. Hence it follows from the inequality

$$
\left\|u_{\mathrm{k}}-z\right\| \leqslant\left\|\mathfrak{u}_{\mathrm{k}}-z_{\mathrm{k}}\right\|+\left\|z_{\mathrm{k}}-z\right\|,
$$

that $u_{k} \rightarrow z$ in $X$. We may suppose that $t_{k} \rightarrow t \in[0,1]$. Since the homotopy $H$ is locally bounded, the sequence $\left(a_{k}\right)$ is clearly bounded, in contradiction to (1.5). Therefore, at least one of the sets $G_{n}$ fulfills (a) and (b), say $G_{n_{0}}=: G_{0}$. This completes the proof.

Moreover, we show that every weakly upper semicontinuous operator of class $\left(S_{+}\right)_{T}$ is proper on bounded closed sets. See [1, Lemma 2.5] and [9, Lemma 2.7] for related single-valued case.

Lemma 1.8. Let $\mathrm{G}$ be a bounded open set in a real reflexive Banach space $\mathrm{X}$ and let $\mathrm{H}:[0,1] \times \overline{\mathrm{G}} \rightarrow 2^{\mathrm{X}}$ be a weakly upper semicontinuous homotopy of class $\left(S_{+}\right)_{T}$, where $T: \bar{G} \rightarrow X^{*}$ is bounded. For each compact set $A$ in $X$,

$$
\mathrm{K}:=\{\mathrm{u} \in \overline{\mathrm{G}} \mid \mathrm{H}(\mathrm{t}, \mathrm{u}) \cap \mathrm{A} \neq \emptyset \text { for some } \mathrm{t} \in[0,1]\},
$$

is a compact set in $\mathrm{X}$.

Proof. Let $A$ be a compact set in $X$. Let $\left(u_{n}\right)$ be any sequence in $K$. Then for every $n \in \mathbb{N}$ we choose $t_{n} \in[0,1]$ and $a_{n} \in A$ such that $a_{n} \in H\left(t_{n}, u_{n}\right)$. Since the set $A$ is compact, there is a subsequence $\left(a_{k}\right)$ of $\left(a_{n}\right)$ such that $a_{k} \rightarrow a$ for some $a \in A$. By the boundedness of the set $G$ and the map $T$, we may suppose, without loss of generality, that

$$
u_{k} \rightarrow u \text { in } X, \quad y_{k}:=T u_{k} \rightarrow y \text { in } X^{*}, \quad \text { and } t_{k} \rightarrow t \text { in }[0,1]
$$

Then we have $\lim _{k \rightarrow \infty}\left\langle a_{k}, y_{k}-y\right\rangle=0$. Since the homotopy $H$ is of class $\left(S_{+}\right)_{T}$ and is weakly upper semicontinuous with weakly closed convex values, this implies that $u_{k} \rightarrow u \in \bar{G}$ and $a \in H(t, u)$. Therefore, we have $u \in K$. Consequently, the set $\mathrm{K}$ is compact in $\mathrm{X}$. This completes the proof. 
Corollary 1.9. Suppose that $\mathrm{F}: \overline{\mathrm{G}} \rightarrow 2^{\mathrm{X}}$ is a locally bounded weakly upper semicontinuous operator of class $\left(\mathrm{S}_{+}\right)_{\mathrm{T}}$, where $\mathrm{G}$ is a bounded open subset of $\mathrm{X}$ and $\mathrm{T}: \overline{\mathrm{G}} \rightarrow \mathrm{X}^{*}$ is bounded. For every $\mathrm{h} \notin \mathrm{F}(\partial \mathrm{G})$, there is an open set $\mathrm{G}_{0}$ in $\mathrm{X}$ such that $\mathrm{F}^{-1}(\mathrm{~h}) \subset \mathrm{G}_{0} \subset \mathrm{G}$ and $\mathrm{F}$ is bounded on $\overline{\mathrm{G}}_{0}$.

Proof. Suppose that $h \notin F(\partial G)$. By Lemma 1.8, $F^{-1}(h)$ is a compact subset of $X$ and $F^{-1}(h) \subset G$. Applying Theorem 1.7 with $S=F^{-1}(h)$, there is an open set $G_{0}$ in $X$ such that $F^{-1}(h) \subset G_{0} \subset G$ and $F$ is bounded on $\overline{\mathrm{G}}_{0}$.

\section{Degree theory}

In this section, $(X,\|\cdot\|)$ will always be an infinite-dimensional real reflexive separable Banach space which has been renormed so that both $X$ and its dual space $X^{*}$ are locally uniformly convex.

In this case, it is known that the duality operator $\mathrm{J}: X \rightarrow X^{*}$ is bijective, bounded, continuous and of class $\left(S_{+}\right)$and such that $\langle J u, u\rangle=\|u\|^{2}$ and $\|J u\|=\|u\|$ for $u \in X$, see e.g. [17].

We first introduce the Berkovits-Tienari degree theory for the class $\mathcal{F}_{S_{+}}(X)$ given in [3] which is based on the known topological degree for Leray-Schauder type set-valued operators in $[7,11]$.

Lemma 2.1. Let $\mathrm{G}$ be any bounded open subset of $\mathrm{X}$ and let $\mathrm{F}: \overline{\mathrm{G}} \rightarrow 2^{\mathrm{X}^{*}}$ be a (locally) bounded weakly upper semicontinuous set-valued operator of class $\left(S_{+}\right)$. If $h \notin F(\partial \mathrm{G})$, then the $\left(S_{+}\right)$-degree of $\mathrm{F}$ on $\mathrm{G}$ over $\mathrm{h}$ is defined as an integer, denoted by $\mathrm{d}_{\mathrm{S}_{+}}(\mathrm{F}, \mathrm{G}, \mathrm{h})$ and the degree has the following properties:

(a) (Existence) If $\mathrm{d}_{\mathrm{S}_{+}}(\mathrm{F}, \mathrm{G}, \mathrm{h}) \neq 0$, then the inclusion $\mathrm{h} \in \mathrm{Fu}$ has a solution in $\mathrm{G}$.

(b) (Additivity) If $\mathrm{G}_{1}$ and $\mathrm{G}_{2}$ are disjoint open subsets of $\mathrm{G}$ such that $\mathrm{h} \notin \mathrm{F}\left(\overline{\mathrm{G}} \backslash\left(\mathrm{G}_{1} \cup \mathrm{G}_{2}\right)\right)$, then we have

$$
d_{S_{+}}(F, G, h)=d_{S_{+}}\left(F, G_{1}, h\right)+d_{S_{+}}\left(F, G_{2}, h\right) .
$$

(c) (Homotopy Invariance) Suppose that $\mathrm{H}:[0,1] \times \overline{\mathrm{G}} \rightarrow 2^{\mathrm{X}^{*}}$ is a (locally) bounded weakly upper semicontinuous homotopy of class $\left(\mathrm{S}_{+}\right)$. If $\mathrm{h}:[0,1] \rightarrow \mathrm{X}^{*}$ is a continuous curve in $\mathrm{X}^{*}$ such that $\mathrm{h}(\mathrm{t}) \notin \mathrm{H}(\mathrm{t}, \mathrm{u})$ for all $(\mathrm{t}, \mathrm{u}) \in[0,1] \times \partial \mathrm{G}$, then the value of $\mathrm{d}_{\mathrm{S}_{+}}(\mathrm{H}(\mathrm{t}, \cdot), \mathrm{G}, \mathrm{h}(\mathrm{t}))$ is constant for all $\mathrm{t} \in[0,1]$.

(d) (Normalization) If $\mathrm{h} \in \mathrm{J}(\mathrm{G})$, then we have $\mathrm{d}_{\mathrm{S}_{+}}(\mathrm{J}, \mathrm{G}, \mathrm{h})=1$.

Next we construct a topological degree for the class $\mathcal{F}_{B}(X)$ with elliptic super-regularization method as in [2]. The idea is basically a compact embedding theorem due to Browder and Ton [5].

Proposition 2.2. Let $\mathrm{Y}$ be a real separable Banach space. Then there exists a separable Hilbert space $\mathrm{W}$ and $a$ compact linear injection $\phi: \mathrm{W} \rightarrow \mathrm{Y}$ such that $\phi(\mathrm{W})$ is dense in $\mathrm{Y}$.

According to Proposition 2.2 with $Y=X^{*}$, let $\phi: W \rightarrow X^{*}$ be a compact linear injection such that $\phi(W)$ is dense in $X^{*}$. We define another operator $\hat{\phi}: X \rightarrow W$ by setting

$$
(\hat{\phi}(v), w)_{W}=\langle v, \phi(w)\rangle, \quad \text { for all } w \in W \text { and } v \in X,
$$

where $(\cdot, \cdot)_{W}$ denotes the inner product of $W$. Obviously, $\hat{\phi}$ is also a compact linear injection.

To each $F \in \mathcal{F}_{\mathrm{T}, \mathrm{B}}$ with $T \in \mathcal{F}_{1}$, we associate a family of operators defined by

$$
F_{\lambda}:=T+\lambda \phi \hat{\phi} F, \quad \text { for } \lambda>0 .
$$

Then each $F_{\lambda}$ is bounded, upper semicontinuous and of class $\left(S_{+}\right)$.

In fact, when constructing a new degree for $F \in \mathcal{F}_{\mathrm{T}}$, we have a great difficulty in applying the above $\left(S_{+}\right)$-degree of $F_{\lambda}$ directly. In view of Theorem 1.7, we proceed in two steps, as we will see below.

We give a fundamental result needed for the construction of a degree and its properties, see [2, Lemma 4.2] for the single-valued case. 
Lemma 2.3. Let $\mathrm{G}$ be any bounded open set in $X$ and $A$ be any closed subset of $\overline{\mathrm{G}}$. Suppose that $\mathrm{F} \in \mathcal{F}_{\mathrm{T}, \mathrm{B}}(\overline{\mathrm{G}})$, where $T \in \mathcal{F}_{1}(\bar{G})$. If $h \notin F(A)$, then there exists a positive number $\lambda_{0}$ such that $h_{\lambda} \notin F_{\lambda}(A)$ for all $\lambda \geqslant \lambda_{0}$, where $h_{\lambda}=\lambda \phi \hat{\phi} h$.

Proof. Let $A$ be a closed subset of $\bar{G}$ such that $h \notin F(A)$. Suppose to the contrary that there are sequences $\left(\lambda_{n}\right)$ in $(0, \infty)$ and $\left(u_{n}\right)$ in $A$ with $\lambda_{n} \rightarrow \infty$ such that $h_{\lambda_{n}} \in F_{\lambda_{n}}\left(u_{n}\right)$, for all $n \in \mathbb{N}$, that is,

$$
T u_{n}+\lambda_{n} \phi \hat{\phi}\left(v_{n}-h\right)=0,
$$

where $v_{n} \in \mathrm{Fu}_{n}$. Passing to subsequences if necessary, we may suppose that

$$
u_{n} \rightarrow u \text { in } X, \quad \text { and } \quad v_{n} \rightarrow v \text { in } X, \quad \text { and } \quad y_{n}:=T u_{n} \rightarrow y \text { in } X^{*} .
$$

Then it follows from (2.2) and the strong continuity of the operator $\phi$ that

$$
\phi \hat{\phi} v_{\mathrm{n}} \rightarrow \phi \hat{\phi} \mathrm{h}=\phi \hat{\phi} v,
$$

which implies $v=\mathrm{h}$, by the injectivity of $\phi \hat{\phi}$. Hence it follows from (2.1), (2.2) and (2.3) that

$$
\begin{aligned}
\limsup _{n \rightarrow \infty}\left\langle v_{n}, y_{n}-y\right\rangle & =\limsup _{n \rightarrow \infty}\left\langle v_{n}-h, y_{n}\right\rangle \\
& =\limsup _{n \rightarrow \infty}\left\langle v_{n}-h,-\lambda_{n} \phi \hat{\phi}\left(v_{n}-h\right)\right\rangle \\
& =\limsup _{n \rightarrow \infty}\left[-\lambda_{n}\left\|\hat{\phi}\left(v_{n}-h\right)\right\|_{W}^{2}\right] \\
& \leqslant 0 .
\end{aligned}
$$

Since $F$ is of class $\left(S_{+}\right)_{T}$ and weakly upper semicontinuous, we have $u_{n} \rightarrow u \in A$ and $h \in F u$, which contradicts the hypothesis that $h \notin F(A)$. This completes the proof.

Corollary 2.4. Suppose that $\mathrm{G}$ is a bounded open set in $\mathrm{X}$ and $\mathrm{F} \in \mathcal{F}_{\mathrm{T}, \mathrm{B}}(\overline{\mathrm{G}})$, where $\mathrm{T} \in \mathcal{F}_{1}(\overline{\mathrm{G}})$. If $\mathrm{h} \notin \mathrm{F}(\partial \mathrm{G})$, there is a positive number $\lambda_{0}$ such that $h_{\lambda} \notin F_{\lambda}(\partial G)$ for all $\lambda \geqslant \lambda_{0}$ and the value of $d_{S_{+}}\left(F_{\lambda}, G, h_{\lambda}\right)$ is constant for all $\lambda \geqslant \lambda_{0}$.

Proof. According to Lemma 2.3 with $A=\partial G$, we can choose a positive number $\lambda_{0}$ such that $h_{\lambda} \notin F_{\lambda}(\partial G)$ for all $\lambda \geqslant \lambda_{0}$. For the second part, let $\lambda_{1}, \lambda_{2}$ be arbitrary in $\left[\lambda_{0}, \infty\right)$ such that $\lambda_{1}<\lambda_{2}$. Then $F_{\lambda}, \lambda \in\left[\lambda_{1}, \lambda_{2}\right]$, defines a bounded upper semicontinuous homotopy of class $\left(S_{+}\right)$such that $h_{\lambda} \notin F_{\lambda}(\partial G)$ for all $\lambda \in\left[\lambda_{1}, \lambda_{2}\right]$. It follows from Lemma 2.1 (c) that

$$
d_{S_{+}}\left(F_{\lambda_{1}}, G, h_{\lambda_{1}}\right)=d_{S_{+}}\left(F_{\lambda_{2}}, G, h_{\lambda_{2}}\right) .
$$

Since $\lambda_{1}, \lambda_{2}$ were arbitrarily chosen in $\left[\lambda_{0}, \infty\right)$, we conclude that the value of $d_{S_{+}}\left(F_{\lambda}, G, h_{\lambda}\right)$ is constant for all $\lambda \geqslant \lambda_{0}$. This completes the proof.

In view of Corollary 2.4, we can now define a topological degree for the class $\mathcal{F}_{\mathrm{B}}(\mathrm{X})$.

Definition 2.5. Let $F \in \mathcal{F}_{T, B}(\bar{G})$, where $G$ is a bounded open set in $X$ and $T \in \mathcal{F}_{1}(\bar{G})$. If $h \notin F(\partial G)$, then we define a topological degree $d_{B}$ by

$$
d_{B}(F, G, h):=\lim _{\lambda \rightarrow \infty} d_{S_{+}}\left(F_{\lambda}, G, h_{\lambda}\right),
$$

where $F_{\lambda}=T+\lambda \phi \hat{\phi} F$ and $h_{\lambda}=\lambda \phi \hat{\phi} h$.

Actually, the value of $d_{B}(F, G, h)$ is independent of the essential inner map $T$ chosen. In the singlevalued case it was proved in [2, Corollary 6.2].

We need the following fact for establishing the homotopy invariance of the degree $d_{B}$. 
Lemma 2.6. Let $\mathrm{G}$ be a bounded open set in $\mathrm{X}$. Suppose that $\mathrm{H}:[0,1] \times \overline{\mathrm{G}} \rightarrow 2^{\mathrm{X}}$ is a bounded weakly upper semicontinuous affine homotopy of class $\left(S_{+}\right)_{T}$ with a common essential inner map $T \in \mathcal{F}_{1}(\overline{\mathrm{G}})$ and that $\mathrm{h}:[0,1] \rightarrow$ $\mathrm{X}$ is a continuous curve in $\mathrm{X}$ such that $\mathrm{h}(\mathrm{t}) \notin \mathrm{H}_{\mathrm{t}}(\partial \mathrm{G})$ for all $\mathrm{t} \in[0,1]$. Then there exists a positive number $\lambda_{0}$ such that

$$
\mathrm{h}_{\lambda}(\mathrm{t}) \notin\left(\mathrm{H}_{\mathrm{t}}\right)_{\lambda}(\partial \mathrm{G}), \quad \text { for all } \mathrm{t} \in[0,1] \text { and all } \lambda \geqslant \lambda_{0},
$$

where $\mathrm{H}_{\mathrm{t}}=\mathrm{H}(\mathrm{t}, \cdot),\left(\mathrm{H}_{\mathrm{t}}\right)_{\lambda}=\mathrm{T}+\lambda \phi \hat{\phi} \mathrm{H}_{\mathrm{t}}$ and $\mathrm{h}_{\lambda}(\mathrm{t})=\lambda \phi \hat{\phi} \mathrm{h}(\mathrm{t})$.

Proof. The proof can be done in a similar way to that of Lemma 2.3.

The degree function $d_{B}$ defined above has the usual basic properties.

Theorem 2.7. Let $\mathrm{G}$ be any bounded open set in $\mathrm{X}$ and suppose that $\mathrm{F} \in \mathcal{F}_{\mathrm{T}, \mathrm{B}}(\overline{\mathrm{G}})$, where $\mathrm{T} \in \mathcal{F}_{1}(\overline{\mathrm{G}})$. Then the following properties are satisfied:

(a) (Existence) If $d_{B}(F, G, h) \neq 0$, then the inclusion $h \in F u$ has a solution in $G$.

(b) (Additivity) If $G_{1}$ and $G_{2}$ are disjoint open subsets of $G$ such that $h \notin F\left(\bar{G} \backslash\left(G_{1} \cup G_{2}\right)\right.$ ), then we have

$$
d_{B}(F, G, h)=d_{B}\left(F, G_{1}, h\right)+d_{B}\left(F, G_{2}, h\right) .
$$

(c) (Homotopy invariance) Suppose that $\mathrm{H}:[0,1] \times \overline{\mathrm{G}} \rightarrow \mathrm{X}$ is a bounded weakly upper semicontinuous affine homotopy of class $\left(S_{+}\right)_{T}$ with the common essential inner map $T$ and that $h:[0,1] \rightarrow X$ is a continuous curve in $X$ such that $h(t) \notin H(t, \partial G)$, for all $t \in[0,1]$. Then the value of $d_{B}(H(t, \cdot), G, h(t))$ is constant for all $t \in[0,1]$.

(d) (Normalization) For any $h \in G$, we have $d_{B}(I, G, h)=+1$.

Proof.

(a): If $h \notin F u$ for all $u \in \bar{G}$, Lemma 2.3 implies that there exists a positive number $\lambda_{0}$ such that $h_{\lambda} \notin F_{\lambda}(\bar{G})$ for all $\lambda \geqslant \lambda_{0}$. It follows from Lemma 2.1 (a) that $d_{S_{+}}\left(F_{\lambda}, G, h_{\lambda}\right)=0$ for all $\lambda \geqslant \lambda_{0}$. By Definition 2.5, we have $d_{B}(F, G, h)=0$.

(b): Applying Lemma 2.3 with $A=\bar{G} \backslash\left(G_{1} \cup G_{2}\right)$, we find a positive number $\lambda_{0}$ such that

$$
h_{\lambda} \notin F_{\lambda}\left(\bar{G} \backslash\left(G_{1} \cup G_{2}\right)\right), \quad \text { for all } \lambda \geqslant \lambda_{0} .
$$

By Lemma 2.1 (b), we have

$$
d_{S_{+}}\left(F_{\lambda}, G, h_{\lambda}\right)=d_{S_{+}}\left(F_{\lambda}, G_{1}, h_{\lambda}\right)+d_{S_{+}}\left(F_{\lambda}, G_{2}, h_{\lambda}\right), \quad \text { for all } \lambda \geqslant \lambda_{0},
$$

which implies

$$
d_{B}(F, G, h)=d_{B}\left(F, G_{1}, h\right)+d_{B}\left(F, G_{2}, h\right) .
$$

(c): In view of Lemma 2.6, we can choose a positive number $\lambda_{0}$ such that

$$
h_{\lambda}(t) \notin\left(H_{t}\right)_{\lambda}(\partial G), \quad \text { for all } t \in[0,1] \text { and all } \lambda \geqslant \lambda_{0} \text {. }
$$

Let $\lambda \in\left(\lambda_{0}, \infty\right)$ be arbitrary but fixed. Then $\tilde{\mathrm{H}}:[0,1] \times \overline{\mathrm{G}} \rightarrow 2^{X^{*}}$ given by

$$
\tilde{H}(t, u):=\left(H_{t}\right)_{\lambda}(u), \quad \text { for }(t, u) \in[0,1] \times \bar{G}
$$

is a bounded upper semicontinuous homotopy of class $\left(S_{+}\right)$such that

$$
h_{\lambda}(t) \notin \tilde{H}(t, u), \quad \text { for all }(t, u) \in[0,1] \times \partial G .
$$

Hence it follows from Lemma 2.1 (c) that $d_{S_{+}}\left(\left(H_{t}\right)_{\lambda}, G, h_{\lambda}(t)\right)$ is constant for all $t \in[0,1]$. For any $t_{1}, t_{2} \in[0,1]$, we have by Definition 2.5 that

$$
\begin{aligned}
d_{B}\left(H\left(t_{1}, \cdot\right), G, h\left(t_{1}\right)\right) & =\lim _{\lambda \rightarrow \infty} d_{S_{+}}\left(\left(H_{t_{1}}\right)_{\lambda}, G, h_{\lambda}\left(t_{1}\right)\right) \\
& =\lim _{\lambda \rightarrow \infty} d_{S_{+}}\left(\left(H_{t_{2}}\right)_{\lambda}, G, h_{\lambda}\left(t_{2}\right)\right) \\
& =d_{B}\left(H\left(t_{2}, \cdot\right), G, h\left(t_{2}\right)\right) .
\end{aligned}
$$


(d): Since the duality operators $\mathrm{J}: \mathrm{X} \rightarrow \mathrm{X}^{*}$ and $\mathrm{J}^{-1}: \mathrm{X}^{*} \rightarrow \mathrm{X}$ are bounded, continuous and of class $\left(\mathrm{S}_{+}\right)$, Lemma $1.4(\mathrm{~b})$ implies that the identity operator $\mathrm{I}=\mathrm{J}^{-1} \circ \mathrm{J}$ belongs to $\mathcal{F}_{\mathrm{J}}(\overline{\mathrm{G}})$. Let $\mathrm{h}$ be any element of $\mathrm{G}$. Choose a positive number $\mathrm{R}$ with $\|\mathrm{h}\|<\mathrm{R}$ such that

$$
d_{B}(I, G, h)=d_{B}\left(I, B_{R}(0), h\right) .
$$

It is clear that $I u \neq t h$, for all $t \in[0,1]$ and $u \in \partial B_{R}(0)$. Taking $h(t)=t h$, for $t \in[0,1]$, we obtain from part (c) that

$$
d_{B}\left(I, B_{R}(0), h\right)=d_{B}\left(I, B_{R}(0), 0\right) .
$$

By Definition 2.5, we have

$$
d_{B}\left(I, B_{R}(0), 0\right)=\lim _{\lambda \rightarrow \infty} d_{S_{+}}\left(I_{\lambda}, B_{R}(0), 0\right),
$$

where $\mathrm{I}_{\lambda}=J+\lambda \phi \hat{\phi} \mathrm{I}$. For sufficiently large $\lambda$, we have

$$
J u+t \lambda \phi \hat{\phi} u \neq 0, \quad \text { for all }(t, u) \in[0,1] \times \partial B_{R}(0),
$$

and hence by Lemma 2.1 (c)

$$
d_{S_{+}}\left(I_{\lambda}, B_{R}(0), 0\right)=d_{S_{+}}\left(J, B_{R}(0), 0\right)=1 .
$$

Therefore we conclude that $d_{B}(I, G, h)=1$. This completes the proof.

Lemma 2.8. Let $F \in \mathcal{F}_{T}(\overline{\mathrm{G}})$, where $\mathrm{G}$ is a bounded open set in $\mathrm{X}$ and $\mathrm{T} \in \mathcal{F}_{1}(\overline{\mathrm{G}})$. Suppose that for $i=1,2, \mathrm{G}_{i}$ is an open subset of $\mathrm{G}$ such that $\mathrm{F}^{-1}(\mathrm{~h}) \subset \mathrm{G}_{i} \subset \mathrm{G}$ and $\mathrm{F}$ is bounded on $\overline{\mathrm{G}}_{i}$. Then we have

$$
d_{B}\left(F, G_{1}, h\right)=d_{B}\left(F, G_{2}, h\right) .
$$

Proof. For $i=1,2$, since $F \in \mathcal{F}_{T, B}\left(\bar{G}_{i}\right)$ and $h \notin F\left(\partial G_{i}\right)$, the degree $d_{B}\left(F, G_{i}, h\right)$ is defined and $F^{-1}(h) \subset$ $\mathrm{G}_{1} \cap \mathrm{G}_{2} \subset \mathrm{G}_{i}$ implies $h \notin F\left(\bar{G}_{i} \backslash\left(\mathrm{G}_{1} \cap \mathrm{G}_{2}\right)\right)$. Then it follows from Theorem 2.7 (b) that

$$
d_{B}\left(F, G_{1}, h\right)=d_{B}\left(F, G_{1} \cap G_{2}, h\right)=d_{B}\left(F, G_{2}, h\right) .
$$

We are now in a position to define a topological degree for the class $\mathcal{F}(X)$ as an extension of the degree $\mathrm{d}_{\mathrm{B}}$ for $\mathcal{F}_{\mathrm{B}}(\mathrm{X})$.

Definition 2.9. Let

$$
M=\left\{(F, G, h) \mid G \in \mathcal{O}, T \in \mathcal{F}_{1}(\bar{G}), F \in \mathcal{F}_{T}(\bar{G}), h \notin F(\partial G)\right\} .
$$

Then we define a degree function $d: M \rightarrow \mathbb{Z}$ as follows:

$$
d(F, G, h):=d_{B}\left(\left.F\right|_{G_{0}}, G_{0}, h\right),
$$

where $G_{0}$ is any open subset of $G$ such that $F^{-1}(h) \subset G_{0}$ and $F$ is bounded on $\bar{G}_{0}$, according to Corollary 1.9. Here, $\left.F\right|_{\bar{G}_{0}}$ denotes the restriction of $F$ to $\bar{G}_{0}$.

In view of Lemma 2.8, the degree $d$ does not depend on the choice of the set $G_{0}$. Especially, if $F$ is bounded on $\bar{G}$, then we may take $G_{0}=G$ and so $d$ and $d_{B}$ coincide on $\mathcal{F}_{T, B}(\bar{G})$.

Finally we present fundamental properties of the degree $d$ for the class $\mathcal{F}(X)$.

Theorem 2.10. Suppose that $\mathrm{F} \in \mathcal{F}_{\mathrm{T}}(\overline{\mathrm{G}})$, where $\mathrm{G}$ is a bounded open set in $\mathrm{X}$ and $\mathrm{T} \in \mathcal{F}_{1}(\overline{\mathrm{G}})$. Then the degree $\mathrm{d}$ has the following properties:

(a) (Existence) If $d(F, G, h) \neq 0$, then the inclusion $h \in F u$ has a solution in $G$. 
(b) (Additivity) If $G_{1}$ and $G_{2}$ are two disjoint open subsets of $G$ such that $h \notin F\left(\bar{G} \backslash\left(G_{1} \cup G_{2}\right)\right)$, then we have

$$
d(F, G, h)=d\left(F, G_{1}, h\right)+d\left(F, G_{2}, h\right) .
$$

(c) (Homotopy invariance) Suppose that $\mathrm{H}:[0,1] \times \overline{\mathrm{G}} \rightarrow \mathrm{X}$ is a locally bounded weakly upper semicontinuous affine homotopy of class $\left(S_{+}\right)_{T}$ with the common essential inner map $T$. If $h:[0,1] \rightarrow X$ is a continuous curve in $X$ such that $h(t) \notin H(t, \partial G)$, for all $t \in[0,1]$, then the value of $d(H(t, \cdot), G, h(t))$ is constant, for all $t \in[0,1]$.

(d) (Normalization) For any $h \in G$, we have $d(I, G, h)=+1$.

Proof. The above four properties follow from the corresponding properties in Theorem 2.7 together with Definition 2.9 and Theorem 1.7.

\section{Application}

In this section, we study the Dirichlet boundary value problem related to the $p$-Laplacian with discontinuous nonlinearity, based on the degree theory in the previous section.

Let $\Omega$ be a bounded domain in $\mathbb{R}^{N}$ with smooth boundary. Let $2<p<N$ and set $p^{\prime}:=p /(p-1)$. We consider the following nonlinear boundary value problem

$$
\left\{\begin{array}{cl}
-\Delta_{\mathfrak{p}} u+u \in-[\underline{g}(x, u), \bar{g}(x, u)], & \text { in } \Omega, \\
u=0, & \text { on } \partial \Omega,
\end{array}\right.
$$

where $\Delta_{\mathfrak{p}}$ is the $\mathrm{p}$-Laplacian given by $\Delta_{\mathrm{p}} u=\operatorname{div}\left(|\nabla u|^{\mathrm{p}-2} \nabla u\right)$ and $g: \Omega \times \mathbb{R} \rightarrow \mathbb{R}$ is a possibly discontinuous function in the sense that

$$
\begin{aligned}
& \underline{g}(x, s)=\liminf _{\eta \rightarrow s} g(x, \eta)=\lim _{\delta \rightarrow 0+} \inf _{|\eta-s|<\delta} g(x, \eta), \\
& \bar{g}(x, s)=\limsup _{\eta \rightarrow s} g(x, \eta)=\lim _{\delta \rightarrow 0+} \sup _{|\eta-s|<\delta} g(x, \eta) .
\end{aligned}
$$

Suppose that $g: \Omega \times \mathbb{R} \rightarrow \mathbb{R}$ is a real-valued function such that

(g1) $\bar{g}$ and $\underline{g}$ are superpositionally measurable, that is, $\bar{g}(\cdot, u(\cdot))$ and $\underline{g}(\cdot, u(\cdot))$ are measurable on $\Omega$ for any measurable function $u: \Omega \rightarrow \mathbb{R}$;

(g2) g satisfies the growth condition:

$$
|g(x, s)| \leqslant k_{0}(x)+c|s|^{q-1}, \quad \text { for almost all } x \in \Omega \text { and all } s \in \mathbb{R},
$$

where $\mathrm{k}_{0} \in \mathrm{L}^{\mathrm{p}^{\prime}}(\Omega), \mathrm{c}$ is a positive constant and $1<\mathrm{q}<\mathrm{p}$.

Let $W_{0}^{1, p}(\Omega)$ be the closure of $C_{0}^{\infty}(\Omega)$ in the Sobolev space

$$
\mathrm{W}^{1, \mathfrak{p}}(\Omega)=\left\{\mathrm{u} \in \mathrm{L}^{\mathrm{p}}(\Omega)|| \nabla \mathrm{u} \mid \in \mathrm{L}^{\mathfrak{p}}(\Omega)\right\},
$$

with the norm

$$
\|\mathrm{u}\|_{1, \mathrm{p}}=\left(\int_{\Omega}|\mathrm{u}|^{\mathrm{p}} \mathrm{d} x+\int_{\Omega}|\nabla \mathrm{u}|^{p} \mathrm{~d} x\right)^{\frac{1}{\mathrm{p}}} .
$$

Due to the Poincare inequality, the norm $\|\cdot\|_{1, p}$ on $W_{0}^{1, p}(\Omega)$ is equivalent to the norm $\|\cdot\|$ given by

$$
\|u\|=\left(\int_{\Omega}|\nabla u|^{p} d x\right)^{\frac{1}{p}}, \quad \text { for } u \in W_{0}^{1, p}(\Omega) .
$$


Note that the Sobolev space $W_{0}^{1, p}(\Omega)$ is a uniformly convex separable Banach space and the canonical embedding $\mathrm{I}: W_{0}^{1, p}(\Omega) \hookrightarrow L^{p}(\Omega)$ is compact, see e.g. [17].

We give some properties of the related operators which will be used later. Recall that $F: X \rightarrow X^{*}$ is uniformly monotone, if there exists a strictly increasing continuous function $\alpha:[0, \infty) \rightarrow[0, \infty)$ with $\alpha(0)=0$ such that $\langle\mathrm{Fu}-\mathrm{Fv}, \mathrm{u}-v\rangle \geqslant \alpha(\|\mathrm{u}-v\|)\|u-v\|$, for all $u, v \in X$.

Lemma 3.1. Let $X=W_{0}^{1, p}(\Omega)$ be the Sobolev space. Then the following statements hold:

(a) The operator $\mathrm{F}: \mathrm{X} \rightarrow \mathrm{X}^{*}$ setting by

$$
\langle\mathrm{Fu}, v\rangle=\int_{\Omega}|\nabla \mathrm{u}|^{\mathrm{p}-2} \nabla \mathrm{u} \cdot \nabla v \mathrm{~d} \mathrm{x}, \quad \text { for } \mathrm{u}, \boldsymbol{v} \in \mathrm{X},
$$

is bounded, continuous and uniformly monotone.

(b) The operator $\mathrm{A}: \mathrm{X} \rightarrow \mathrm{X}^{*}$ setting by

$$
\langle A u, v\rangle=\int_{\Omega} u v d x, \quad \text { for } u, v \in X
$$

is compact.

(c) Under (g1) and (g2), the set-valued operator $\mathrm{N}: \mathrm{X} \rightarrow 2^{\mathrm{X}^{*}}$ setting by

$$
\begin{aligned}
\mathrm{Nu}=\left\{z \in X^{*} \mid \text { there exists a function } w \in \mathrm{L}^{\mathrm{p}^{\prime}}(\Omega)\right. \text { such that } \\
\\
\quad \underline{\mathrm{g}}(\mathrm{x}, \mathrm{u}(\mathrm{x})) \leqslant \mathrm{w}(\mathrm{x}) \leqslant \overline{\mathrm{g}}(\mathrm{x}, \mathrm{u}(\mathrm{x})) \text { for almost all } \mathrm{x} \in \Omega \\
\text { and } \left.\langle z, v\rangle=\int_{\Omega} w v \mathrm{~d} x \text { for all } v \in X\right\},
\end{aligned}
$$

is bounded, upper semicontinuous, compact and $\mathrm{Nu}$ is nonempty, closed and convex for every $\mathrm{u} \in \mathrm{X}$.

Proof. Since the canonical linear embedding $I: X \hookrightarrow L^{p}(\Omega)$ is compact, it is known in e.g., [13, Theorem 4.19] that the adjoint operator $I^{*}: \mathrm{L}^{\mathrm{p}^{\prime}}(\Omega) \rightarrow \mathrm{X}^{*}$ is compact and moreover, strongly continuous.

(a) It can be found in e.g., [17, Proposition 26.10].

(b) Since the embedding $i: L^{p}(\Omega) \hookrightarrow L^{p^{\prime}}(\Omega)$ is continuous, it follows that $A=I^{*} \circ i \circ I$ is compact.

(c) The set-valued operator $\Phi: \mathrm{L}^{\mathrm{p}}(\Omega) \rightarrow 2^{\mathrm{L}^{\mathrm{p}^{\prime}}(\Omega)}$ given by

$$
\Phi u=\left\{w \in L^{p^{\prime}}(\Omega) \mid \underline{g}(x, u(x)) \leqslant w(x) \leqslant \bar{g}(x, u(x)) \text { for almost all } x \in \Omega\right\}
$$

is bounded and upper semicontinuous and has weakly precompact convex values, see [6, Theorem 1.1].

Hence $\mathrm{N}=\mathrm{I}^{*} \circ \Phi \circ \mathrm{I}$ is obviously bounded, upper semicontinuous and compact.

Definition 3.2. A point $u \in W_{0}^{1, p}(\Omega)$ is said to be a weak solution of (3.1), if there exists a point $z \in N u$ such that

$$
\int_{\Omega}|\nabla u|^{p-2} \nabla u \cdot \nabla v \mathrm{~d} x+\int_{\Omega} u v d x+\langle z, v\rangle=0 \quad \text { for all } v \in \mathrm{W}_{0}^{1, p}(\Omega) .
$$

Now we prove that the given elliptic problem (3.1) has a weak solution. For this, we use the known Browder-Minty theorem on monotone operators and the degree theory.

Theorem 3.3. Under conditions (g1) and (g2), (3.1) has a weak solution $u$ in $\mathrm{W}_{0}^{1, \mathrm{p}}(\Omega)$. 
Proof. Let $\mathrm{Y}=\mathrm{W}_{0}^{1, \mathrm{p}}(\Omega)$ be the Sobolev space and let $\mathrm{F}, \mathrm{A}: \mathrm{Y} \rightarrow \mathrm{Y}^{*}$ and $\mathrm{N}: \mathrm{Y} \rightarrow 2^{\mathrm{Y}^{*}}$ be defined as in Lemma 3.1. Then $u \in Y$ is a weak solution of (3.1), if and only if

$$
\mathrm{Fu} \in-(\mathrm{A}+\mathrm{N}) \mathrm{u} \text {. }
$$

Notice by Lemma 3.1 that the operator $F: Y \rightarrow Y^{*}$ is bounded, continuous and uniformly monotone and in particular, coercive and of class $\left(S_{+}\right)$and the operator $S:=A+N: Y \rightarrow 2^{Y^{*}}$ is bounded, upper semicontinuous and quasimonotone. Now let $X=Y^{*}$ and identify $X^{*}$ with $Y$. By the Browder-Minty theorem on monotone operators in [17, Theorem 26.A], the inverse operator $T:=F^{-1}: X \rightarrow X^{*}$ is bounded, continuous and of class $\left(S_{+}\right)$, where the last follows from the fact that $F$ is continuous and of class $\left(S_{+}\right)$ and $\mathrm{T}$ is bounded. Consequently, (3.3) can be written equivalently as

$$
u=\mathrm{T} v, \quad \text { and } \quad v \in-\mathrm{ST} v .
$$

To solve (3.4), we will employ the degree theory for $\mathcal{F}(X)$. To do this, we first show that the set

$$
\mathrm{B}:=\{v \in \mathrm{X} \mid v \in-\mathrm{tST} v \text { for some } \mathrm{t} \in[0,1]\},
$$

is bounded in $X$. Indeed, let $v \in B$, that is, $v+t a=0$ for some $t \in[0,1]$, where $a \in S T v$. Setting $\mathrm{u}:=\mathrm{T} v$, we write $\mathrm{a}=\mathrm{Au}+z \in \mathrm{Su}$, where $z \in \mathrm{Nu}$, that is, $\langle z, \mathfrak{u}\rangle=\int_{\Omega} w u d x$, for some $w \in \mathrm{L}^{\mathfrak{p}^{\prime}}(\Omega)$ with $g(x, u(x)) \leqslant w(x) \leqslant \bar{g}(x, u(x))$ for almost all $x \in \Omega$. Noting that the embeddings $L^{p}(\Omega) \hookrightarrow L^{2}(\Omega)$, $\mathrm{L}^{\mathrm{p}}(\Omega) \hookrightarrow \mathrm{L}^{\mathrm{q}}(\Omega)$ and $\mathrm{Y} \hookrightarrow \mathrm{L}^{\mathrm{p}}(\Omega)$ are continuous, we get by (g2) the estimate

$$
\begin{aligned}
\|\mathrm{T} v\|^{\mathrm{p}} & =\langle v, \mathrm{~T} v\rangle=-\mathrm{t}\langle\mathrm{a}, \mathrm{T} v\rangle=-\mathrm{t} \int_{\Omega}(u+w) \mathrm{udx} \\
& \leqslant \operatorname{const}\left(\|\mathrm{T} v\|^{2}+\|\mathrm{T} v\|^{\mathrm{q}}+\|\mathrm{T} v\|\right),
\end{aligned}
$$

where $\|\cdot\|$ denotes the equivalent norm on $Y$ given by (3.2). Hence it is obvious that

$$
\{\mathrm{T} v \mid v \in \mathrm{B}\} \text { is bounded in } \mathrm{Y} \text {. }
$$

Since the operator $S$ is bounded, it follows from (3.4) that the set B is bounded in X. We can now choose a positive constant $R$ such that

$$
\|v\|_{\mathrm{X}}<\mathrm{R}, \quad \text { for all } v \in \mathrm{B} \text {. }
$$

This says that

$$
v \notin-\mathrm{tST} v, \quad \text { for all } v \in \mathrm{\partial B}_{\mathrm{R}}(0) \text { and all } \mathrm{t} \in[0,1] \text {. }
$$

Lemma 1.4 implies that

$$
\mathrm{I}+\mathrm{ST} \in \mathcal{F}_{\mathrm{T}}\left(\overline{\mathrm{B}_{\mathrm{R}}(0)}\right) \text {, and } \mathrm{I}=\mathrm{FT} \in \mathcal{F}_{\mathrm{T}}\left(\overline{\mathrm{B}_{\mathrm{R}}(0)}\right) .
$$

Consider an affine homotopy $\mathrm{H}:[0,1] \times \overline{\mathrm{B}_{\mathrm{R}}(0)} \rightarrow 2^{\mathrm{X}}$ given by

$$
H(t, v):=(1-t) I v+t(I+S T) v, \quad \text { for }(t, v) \in[0,1] \times \overline{B_{R}(0)}
$$

Applying Theorem 2.10, we get

$$
\mathrm{d}\left(\mathrm{I}+\mathrm{ST}, \mathrm{B}_{\mathrm{R}}(0), 0\right)=\mathrm{d}\left(\mathrm{I}, \mathrm{B}_{\mathrm{R}}(0), 0\right)=1,
$$

and hence there exists a point $v \in \mathrm{B}_{\mathrm{R}}(0)$ such that

$$
v \in-\mathrm{ST} v,
$$

which says that $u=T v$ is a solution of (3.3). We conclude that $u$ is a weak solution of (3.1). This completes the proof. 


\section{Acknowledgment}

This work was supported by Sungkyun Research Fund, Sungkyunkwan University, 2014.

\section{References}

[1] J. Berkovits, On the degree theory for nonlinear mappings of monotone type, Ann. Acad. Sci. Fenn. Ser. A I Math. Dissertationes, 58 (1986), 58 pages. 1

[2] J. Berkovits, Extension of the Leray-Schauder degree for abstract Hammerstein type mappings, J. Differential Equations, 234 (2007), 289-310. 1, 1, 1, 1, 2, 2, 2

[3] J. Berkovits, M. Tienari, Topological degree theory for some classes of multis with applications to hyperbolic and elliptic problems involving discontinuous nonlinearities, Dynam. Systems Appl., 5 (1996), 1-18. 1, 1, 2

[4] F. E. Browder, Fixed point theory and nonlinear problems, Bull. Amer. Math. Soc. (N.S.), 9 (1983), 1-39. 1

[5] F. E. Browder, B. A. Ton, Nonlinear functional equations in Banach spaces and elliptic super-regularization, Math. Z., 105 (1968), 177-195. 2

[6] K.-C. Chang, The obstacle problem and partial differential equations with discontinuous nonlinearities, Comm. Pure Appl. Math., 33 (1980), 117-146. 3

[7] A. Granas, Sur la notion du degré topologique pour une certaine classe de transformations multivalentes dans les espaces de Banach, (French) Bull. Acad. Polon. Sci. Sér. Sci. Math. Astr. Phys., 7 (1959), 191-194. 1, 2

[8] I.-S. Kim, J.-H. Bae, Elliptic boundary value problems with discontinuous nonlinearities, J. Nonlinear Convex Anal., 17 (2016), 27-38. 1

[9] I.-S. Kim, S.-J. Hong, A topological degree for operators of generalized ( $s_{+}$) type, Fixed Point Theory Appl., 2015 (2015), 16 pages. $1,1,1,1,1$

[10] J. Leray, J. Schauder, Topologie et équations fonctionnelles, Ann. Sci. Éc. Norm., 51 (1934), 45-78. 1

[11] T.-W. Ma, Topological degrees of set-valued compact fields in locally convex spaces, Dissertationes Math. Rozprawy Mat., 92 (1972), 43 pages. 1, 2

[12] D. O'Regan, Y. J. Cho, Y.-Q. Chen, Topological degree theory and applications, Series in Mathematical Analysis and Applications, Chapman \& Hall/CRC, Boca Raton, FL, (2006). 1

[13] W. Rudin, Functional analysis, Second edition, International Series in Pure and Applied Mathematics, McGrawHill, Inc., New York, (1991). 3

[14] I. V. Skrypnik, Nonlinear elliptic equations of higher order, (Russian) Gamoqeneb. Math. Inst. Sem. Mosen. Anotacie., 7 (1973), 51-52. 1

[15] I. V. Skrypnik, Methods for analysis of nonlinear elliptic boundary value problems, Translated from the 1990 Russian original by Dan D. Pascali, Translations of Mathematical Monographs, American Mathematical Society, Providence, RI, (1994). 1

[16] E. Zeidler, Nonlinear functional analysis and its applications, I, Fixed-point theorems, Translated from the German by Peter R. Wadsack, Springer-Verlag, New York, (1985). 1

[17] E. Zeidler, Nonlinear functional analysis and its applications, II/B, Nonlinear monotone operators, Translated from the German by the author and Leo F. Boron, Springer-Verlag, New York, (1990). 2, 3, 3, 3 